\begin{tabular}{|l|l|c|c|}
\hline Eiszeitalter u. Gegenwart & $\mathbf{2 8}$ & $\begin{array}{c}119-125 \\
6 \mathrm{Abb} .\end{array}$ & Öbringen/Württ. 1978 \\
\hline
\end{tabular}

\title{
Glazialtektonik und Glazialstratigraphie, mit Beispielen aus Dänemark*
}

\author{
STEEN SJöRRING **) \\ Glacial dislocations, glacial stratigraphy, sediment petrology, Denmark
}

Kurzfassung: In Dänemark sind eine Reihe quartärstratigraphischer Untersuchungen mit Hilfe glazialtektonischer Studien durchgeführt worden. Einige Beispiele veranschaulichen die engen Beziehungen zwischen der glazialtektonischen Lagerung und der Quartärstratigraphie.

\section{[Glacial Dislocations and Glacial Stratigraphy Illustrated by Examples from Denmark]}

A bstract: The close relationship between studies of glacial disturbances and the establishment of a local glacial stratigraphy is proved and illustrated by some selected outcrops from Denmark.

\section{Einleitung}

Die Aufschlußuntersuchungen der letzten Jahre in Dänemark haben die Diskussion über die Glazialstratigraphie in Bewegung gebracht. Die Ursache liegt darin, daß die neuen Untersuchungen und die daraus folgenden Ergebnisse nicht mit den klassischen Auffassungen (HANSEN 1965) über den Verlauf der Eiszeiten übereinstimmen. Dazu kommen in letzter Zeit einige neue Gesichtspunkte, die fraglos eine Vereinfachung der Eiszeitgeologie ergeben (MARCUSSEN 1977).

Die größte Uneinigkeit besteht über die Verhältnisse während des Weichsel-Glazials. Sie kommt zum Ausdruck in der verschiedenen Einstellung mancher Autoren zu denselben Beobachtungen: Eine schwach geschichtete Moräne könnte als „Flow-till“ oder als Grundmoräne mit typischer „glaziodynamischer Struktur“ gedeutet werden, und ein Faltungskomplex könnte entweder als Ergebnis glazialtektonischer Vorgänge oder als Folge des Toteisabbaues ("slumping “) erklärt werden.

In diesem Referat werden einige glazialtektonische Beispiele vorgelegt, die zu der neuen Ansicht über die Glazialstratigraphie in Dänemark geführt haben.

\section{Die Untersuchungsergebnisse und ihre Interpretation}

Seit mehr als 100 Jahren sind die glazialtektonischen Verhältnisse in Dänemark — besonders an den Steilufern - untersucht worden. Eine Reihe von klassischen Untersuchungen wurde am Ristinge Klint auf Langeland durchgeführt (Abb. 1); es seien hier nur die Untersuchungen von MAdSEn \& Nordmann \& HARTz (1908), MAdSen (1916) und RosenKRANTZ (1944) erwähnt. Eine Übersicht auf Deutsch gab MadSEn (1928 a, 1928 b).

Auf der Skizze von Ristinge Klint (Abb. 2, oberer Teil) sieht man, daß die Schichtenfolge aus sieben Einheiten zusammengesetzt ist. Davon sind die sechs älteren Einheiten verschuppt, und nur die jüngste Einheit, die diskordante Moräne, ist nicht gestört worden.

*) Vortrag auf der Regionalkonferenz der GLQD-INQUA-Kommission am 17. 10. 1977 in Hamburg. Für redaktionelle Unterstützung danke ich F. Grube und vor allem Herrn J. Ehlers.

$*$ ) Anschrift des Verfassers: Universitätslektor Steen Sjörring, Institut for Almen Geologi, Københavns Universitet, Østervoldgade 10, DK-1350 København K, Dänemark. 
Deshalb scheint ein Zusammenhang zwischen dem Eis, das die Schichtenfolge gestört hat, und der Ablagerung der diskordanten Moräne durchaus logisch.

Die Kiesanalyse der Fraktion 1,41-6 mm in der diskordanten Moräne (BAHNSON 1973) ergab einen Quotienten paläozoischer Kalksteine und Schiefer zu magmatischen und metamorphen Gesteinen von bis zu 0,6 (ein relativ hoher Wert). Weil die paläozoischen Gesteine aus dem baltischen Raum stammen und die Störung der Schichten von Südosten her erfolgt ist, ist die diskordante Moräne allem Anscheinen nach dem jungbaltischen Eisstrom (Eisrandlinie $\mathrm{W}_{\mathbf{3}}$ auf $\mathrm{Abb} .1$ ) zuzuordnen.

Innerhalb der älteren Einheiten des Kliffs finden sich zwei weitere weichselzeitliche Moränen, die aufgrund ihrer unterschiedlichen Petrographie zwei verschiedenen Eisvorstößen angehören müssen. Die untere Moräne (die „dünne Moräne“ nach MADSEN 1928 b) hat einen $\mathrm{P} / \mathrm{K}$-Quotienten von 0,8 und muß deshalb auch aus dem baltischen Raum nach Ristinge gebracht worden sein. Die obere gestauchte Moräne (die "mächtige Moräne" nach

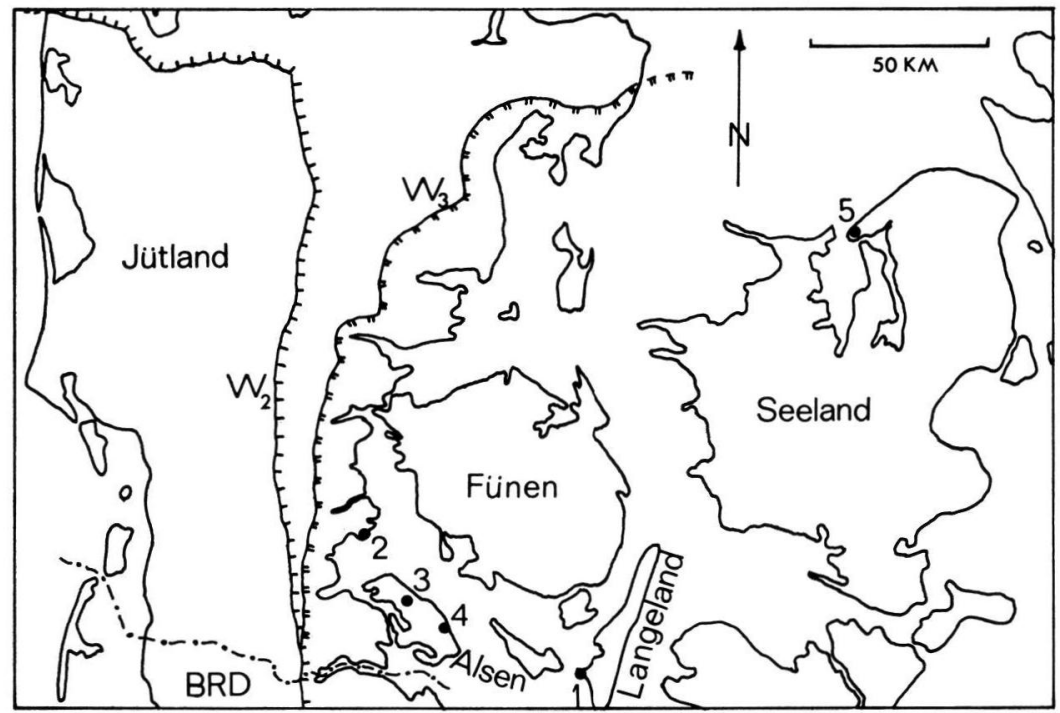

Abb. 1: Lage der erwähnten Lokalitäten. 1. Ristinge Kliff, 2. Halk Hoved Kliff, 3. Kiesgrube Hundslev, 4. Sarup Kliff, 5. Hundested Kliff. $-\mathrm{W}_{2}=$ Eisrandlinie des Nordost-Eises, $\mathrm{W}_{3}=$ Eisrandlinie des jungbaltischen Eisstromes.

MAdsen 1928 b) enthält ein Geschiebepflaster mit einer Schrammenrichtung, die von Nordost nach Südwest weist. Sie hat auch nur einen $\mathrm{P} / \mathrm{K}$-Quotienten von 0,3 und dürfte daher - in Ubereinstimmung mit der Schrammenrichtung - wahrscheinlich aus Nordosten herantransportiert worden sein (ANDERSEN 1950, BERTHELSEN 1973 und SJøRRING 1977 a). Diese Moräne ist daher wahrscheinlich dem Nordost-Eisstrom (Eisrandlage $W_{2}$ in Abb. 1) zuzuordnen.

Ähnlich wie im Kliff bei Ristinge gibt es auch im Steilufer bei Halk Hoved (Abb. 1) drei verschiedene Moränen (Jessen 1930 und Frederiksen 1976). Bei Halk ist aber der Zeitpunkt der Dislokation früher anzusetzen als bei Ristinge und zeitlich mit derAblagerung der mittleren Moräne gleichzusetzen (Abb. 2, unterer Teil). Im Hinblick auf ihre Petrographie und die strukturellen Verhältnisse ist die mittlere Moräne dem NordostEisstrom zuzuordnen (Eisrandlage $\mathrm{W}_{2}$ in $\mathrm{Abb}$. 1), und die obere Moräne gehört zum jungbaltischen Eisvorstoß (Eisrandlage $\mathrm{W}_{3}$ in Abb. 1). Die untere Moräne im Kliff könnte da- 


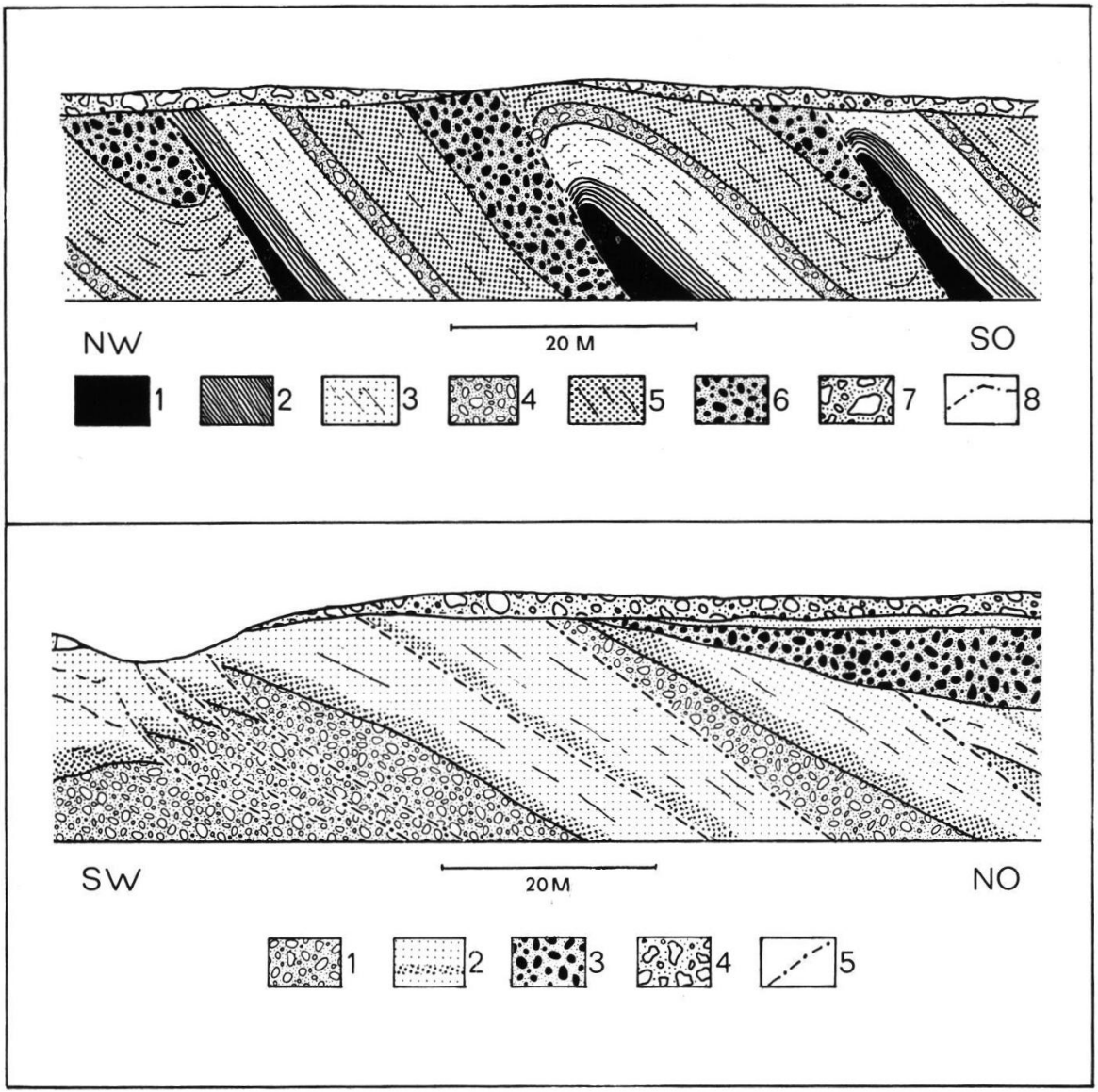

Abb. 2. O b e n : Ristinge Kliff. 1. Saalezeitlicher Ton (der „blanke“ Ton), 2. Eem-Ablagerungen, 3. Glaziofluvialer Sand, 4. Baltische Moräne (die „dünne Moräne"), 5. Glaziofluvialer Sand, 6. Nordost-Moräne (die "mächtige Moräne“), 7. Baltische Moräne (die diskordante Moräne), 8. Überschiebungen. - Unte n : Halk Hoved Kliff. 1. Die untere Moräne, 2. Glaziofluviale Sand- und Kiesablagerungen, 3. Die mittlere Moräne (Nordost-Moräne), 4. Die obere Moräne (Baltische Moräne), 5. Überschiebungen.

gegen entweder saalezeitlich oder früh-weichselzeitlich abgelagert worden sein. Nach den Untersuchungen von FrEDERIKSEN (1976) und nach einigen Feinkiesanalysen (Zählungen von J. EHLERs, Universität Hamburg), ist die untere Moräne wahrscheinlich in die SaaleEiszeit einzustufen.

$\mathrm{Da}$ die mittlere Moräne stellenweise im Steilufer fehlt, sind die tektonischen und stratigraphischen Verhältnisse nicht immer eindeutig zu erkennen, wenn man nicht auch die Umgebung der kritischen Stellen genau untersucht hat. Zum Beispiel könnte der in der südwestlichen Hälfte von Abb. 2 (unten) gezeigte Ausschnitt zu der Annahme verleiten, daß die obere Moräne zu dem Eisvorstoß gehört, der die Schichtenfolge gestört hat.

Dies Beispiel beweist, daß oberflächliche Untersuchungen oft $\mathrm{zu}$ falschen Deutungen führen können. Um diesen Schwierigkeiten beizukommen, muß man die sedimentstrukturellen und tektonischen Verhältnisse so genau wie möglich untersuchen. Einen wichtigen 
Hinweis kann z. B. die Schüttungsrichtung der Schmelzwassersande geben. Für den Fall, daß die Fließrichtung in den Schmelzwasserablagerungen mit der ursprünglichen Bewegungsrichtung in der überliegenden (oder unterliegenden) Moräne übereinstimmt, liegt es nahe, daß die Schmelzwasserablagerung und die Moräne zum selben Eisvorstoß gehören. Stehen dagegen die zwei Bewegungsrichtungen im Widerspruch, muß es sich um zwei verschiedene Phasen handeln. Aufgrund dieser Betrachtungsweise ist es möglich, die Schichten $\mathrm{zu}$ mehreren übergeordneten Einheiten zusammenzustellen - den sogenannten „kinetostratigraphischen Einheiten“ (BERTHELSEN 1973 und SJøRRING 1977 b). Zu jeder Einheit sollte jeweils ein Eisvorstoß gehören.

Die Erfahrung zeigt, daß in der Natur selten Idealprofile ausgebildet sind. Sehr oft fehlen in der Schichtenfolge eine oder mehrere Einheiten. Deshalb kann es besonders in einem Wandprofil ohne glazialtektonische Störungen schwer sein, eine korrekte Deutunz vorzunehmen. Das fällt leichter im Fall von gestörten Ablagerungen, zumal in diesen Fällen oft auch ältere Einheiten mit emporgepreßt worden sind (z. B. warmzeitliche Ablagerungen, die stratigraphisch gut einzuordnen sind).

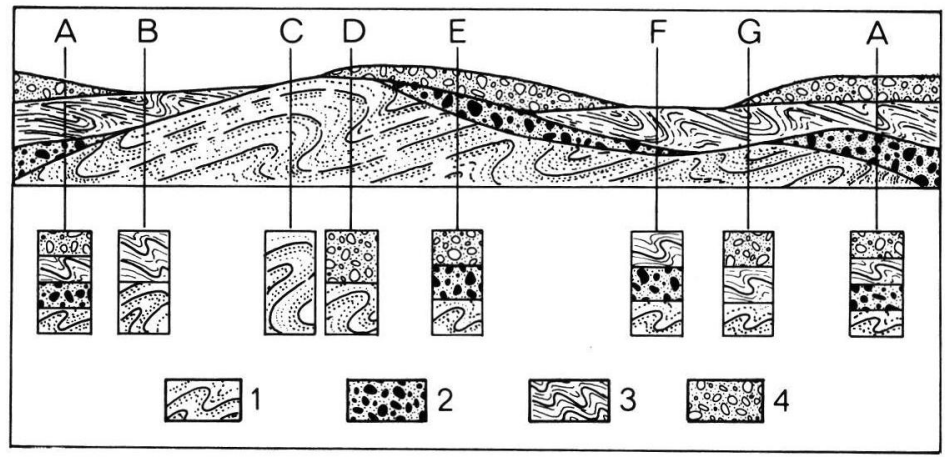

Abb. 3: Schematisches Profil mit zwei tektonischen Stockwerken. 1. Schmelzwassersedimente, von links gefaltet, 2. Moräne, von links abgelagert, 3. Schmelzwassersedimente, von rechts gefaltet, 4. Moräne, von rechts abgelagert.

In Abb. 3 ist ein Gedankenexperiment gemacht worden. Wir gehen davon aus, daß das mit "A" bezeichnete Profil eine vollständige Folge von vier verschiedenen Schichten darstellt. In den übrigen Säulen fehlen jeweils eine oder mehrere Formationen, so daß die korrekte Deutung erschwert ist. Profil D zeigt übrigens die erwähnten Verhältnisse in der südwestlichen Hälfte vom Halk Hoved Kliff (Abb. 2 unten). Die Interpretation einzelner Bohrungen oder kleiner Aufschlüsse kann daher leicht zu Fehldeutungen führen.

Die allgemeinen Formen der Störungen sind als Ergebnis der Falten- oder Schollentektonik zu sehen. Oft treten reverse Verwerfungen oder Überschiebungen auf, deren Neigungsrichtung gegen die Eisbewegungsrichtung weist. Gelegentlich kann es allerdings schwer sein, Festzustellen, ob es sich um reverse oder normale Verwerfungen innerhalb der gestörten Schollen handelt. In Abb. 4 (Ostküste von Alsen, Abb. 1) werden normale Verwerfungen in ungestörter Schichtenfolge (A) als reverse Verwerfungen erscheinen, wenn die Schichten senkrecht gestellt worden sind (B); im Gegensatz dazu werden ursprünglich reverse Verwerfungen (C) bei Senkrechtstellung zu normalen Verwerfungen verändert (D). Hier muß man zunächst das Alter der Verwerfungen (früher oder später als die Kippung der Schichten) bestimmen, wenn man die geologische Geschichte erklären will. 


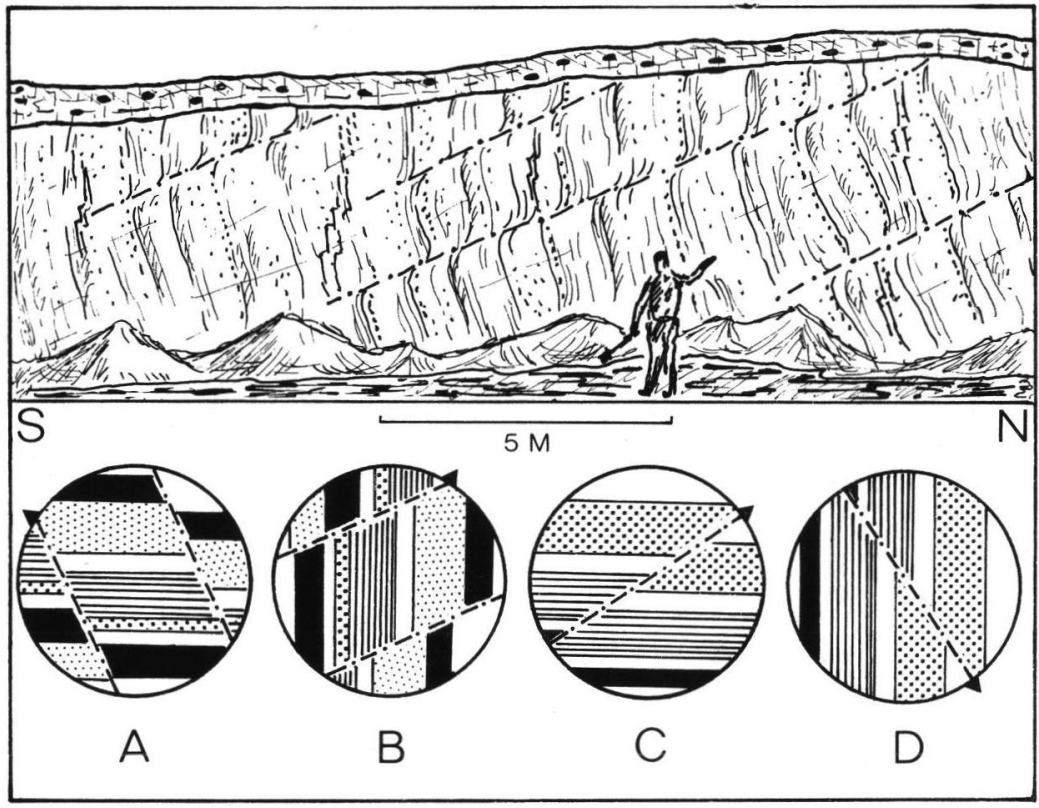

Abb. 4: Ostküste von Alsen bei Sarup. Gestauchte Schmelzwassersedimente, mit einer baltischen Moräne überdeckt. Erläuterungen siehe Text.

Bei den Faltungen ergibt sich ein anderes Problem. Betrachten wir als Beispiel die Kiesgrube von Hundslev auf Alsen (Abb. 1). Die Schichtenfolge wird von Schmelzwassersanden aufgebaut (Abb. 5), die stark gefaltet worden sind. Die Deutung ist aber nicht ohne Probleme, weil als Erklärung zwei geometrische Möglichkeiten vorhanden sind: Entweder könnte die Falte durch Eisdruck aus Westen (Abb. $5 \mathrm{D}_{\mathrm{A}}$ ) - oder durch Fisdruck aus Osten (Abb. $5 \mathrm{E}_{\mathrm{D}}$ ) geschaffen worden sein. Hier muß erst die ursprüngliche Oberseite der Schichten gefunden werden, bevor die Vorstoßrichtung des stauchenden Eises festgestellt werden kann. In diesem Fall ließ sich auf Grund der überkippten Schrägschichtungskörper feststellen, daß der Eisdruck aus Osten gekommen ist.

Abb. 5 A zeigt zwei Skizzen von der Kiesgrube in den Jahren 1974 und 1975. Aus der Gestalt der Schichten ist nicht ohne weiteres zu erkennen, daß es sich in beiden Fällen um dieselbe Struktur handelt. Die Ursache dafür ist in der schwach nach Norden einfallenden Faltenachse zu suchen (Abb. 5 B). Deshalb ist es wichtig, daß man die tektonischen Strukturen in der Richtung der Faltenachse besieht (Abb. 5 C). Falls der Kiesabbau weitergeht, werden von der Struktur in ein paar Jahren nur noch flachliegende Schichten zu sehen sein. Diese Schichten liegen aber in Wirklichkeit mit der Unterseite nach oben.

Bei den bisher gezeigten Beispielen liegt jeweils nur ein tektonisches Stockwerk vor. An mehreren Stellen treten jedoch auch Störungen auf, die von mehreren verschiedenen Eisströmen erzeugt wurden. Als Beispiel sei die Steilküste bei Hundested (Abb. 1) erwähnt. Im basalen Teil des Profils (Abb. 6) findet man eine weichselzeitliche Grundmoräne, die von Schmelzwassersedimenten überlagert ist (SJøRRING 1973, 1974). Die erwähnte Moräne und die zugehörigen Schmelzwassersande sind aus nördlicher Richtung zusammen gefaltet worden. Das ist nicht ungewöhnlich, doch folgen über diesem gestauchten Komplex weitere Schmelzwassersedimente, die nicht mit den Faltungen in Zusammenhang stehen. Das bedeutet also, daß zwischen den beiden Schmelzwasserablagerungen ein Eisvorstoß stattgefunden haben muß, ohne daß Moränenablagerungen davon zu finden sind. 


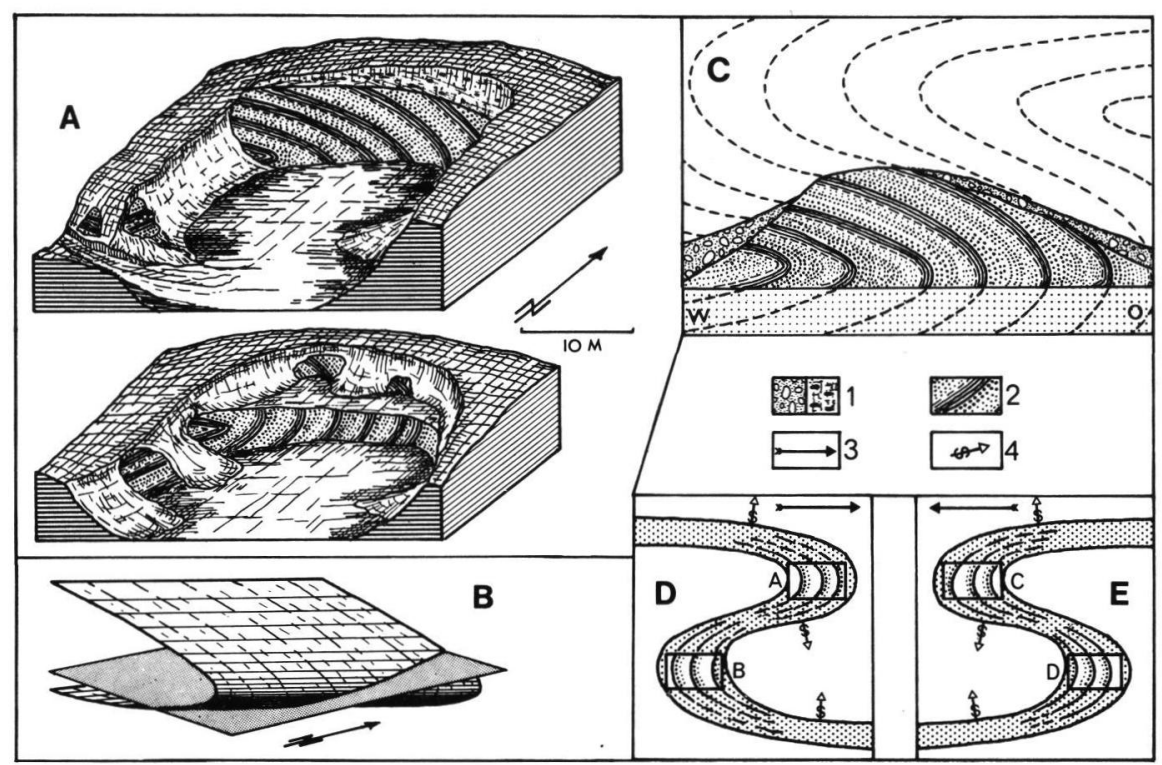

Abb. 5: Kiesgrube Hundslev, Alsen. A. Skizzen aus der Grube in den Jahren 1974 (unten) und 1975 (oben), B. Schematische Darstellung der nach Norden einfallenden Faltenachse, C. Die glaziotektonische Struktur in Achsenrichtung gesehen, D. Geometrische Darstellungen der Deutungsmöglichkeiten; 1. Tonige Moräne, 2. Schmelzwasserablagerungen, 3. Eisbewegungsrichtung (in D und E), 4. Oberseite der Schichten (in D und E).

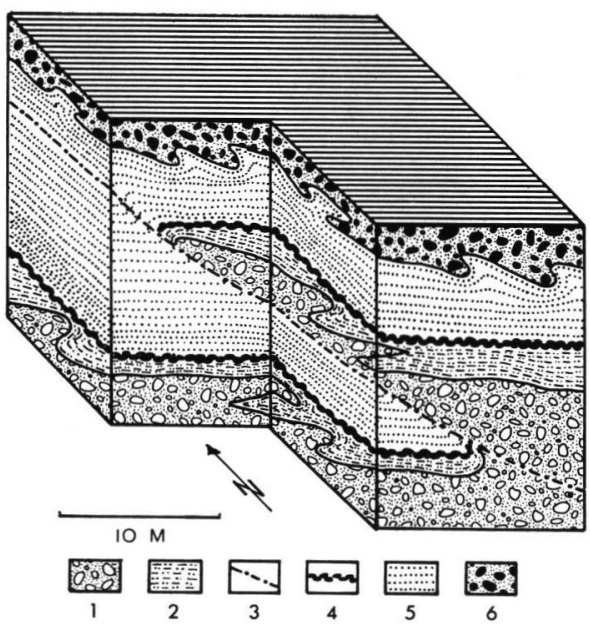

Abb. 6: Hundested Kliff. 1. Untere Moräne, 2. Schmelzwasserablagerungen des unteren Stockwerkes, 3. Überschiebungen, 4. Kies- und Steinband, 5. Schmelzwasserablagerungen des oberen Stockwerkes, 6. Obere Moräne. 
Die oberen Schmelzwassersedimente sind als proglaziale Sanderablagerungen gedeutet worden. Sie sind später von dem vordringenden Eis zusammen mit der zugehörigen Grundmoräne aus östlicher Richtung gefaltet worden (Abb. 3 B und G). Bei dieser letzten Stauchung sind auch reverse Verwerfungen gebildet worden, an denen Teile des unteren Stockwerks nach oben geschoben worden sind, so daß man heute ein Kliff mit drei Moränen sieht. Die zwei unteren Moränen sind jedoch in Wirklichkeit identisch. In struktureller Hinsicht sind aber die "mittlere" und de obere Moräne praktisch gleichzeitig in ihre heutige Position gebracht worden, wodurch die "mittlere“ Moräne hier auf jüngeren Schmelzwasserablagerungen ruht.

Diese wenigen Beispiele aus Dänemark mögen zeigen, mit welchen glazialtektonischen und -stratigraphischen Problemen bei Aufschlußuntersuchungen gerechnet werden muß. Die Geländearbeit in den kommenden Jahren wird sicher weitere Ergebnisse bringen. Nach den Untersuchungen der letzten Jahre läßt sich jedoch sagen, daß eine Glazialstratigraphie ohne glazialtektonische Studien wohl kaum auskommen kann, wenn man nicht eine allzu grobe Vereinfachung in Kauf nehmen will.

\section{Schriftenverzeichnis}

Andersen, S. A. (1950): Raageleje egnens geologi. - Meddr. dansk geol. Foren., 11: 543-557, $1 \mathrm{Abb}$; (English abstract: A re-interpretation of certain glacial morphological features of the northeast of Zealand, Denmark.); Kopenhagen (Reitzel).

BaHnson, H. (1973): Lithological investigations in some danish boulder-clay profiles. - Bull. geol. Inst. Univ. Uppsala, N.S., 5: 93-109, 13 Abb., 2 Tab.; Stockholm (Almqvist \& Wiksell).

Berthelsen, A. (1973): Weichselian ice advances and drift successions in Denmark. - Bull. geol. Inst. Univ. Uppsala, N.S., 5: 21-29, 2 Abb.; Stockholm (Almqvist \& Wiksell).

Frederiksen, J. (1976): Hvad soenderjyske klinter fortaeller. - VARV, 2: 35-45, 7 Abb.; Kopenhagen.

HAnsen, S. (1965): The Quaternary of Denmark. - In: Rankama, K. (Hrsg.): The Quaternary, 1: 90 S., 16 Abb., 18 Tab.; New York - London - Sidney (Interscience Publishers).

Jessen, A. (1930): Klinten ved Halkhoved. - Danm. geol. Unders. Rk. IV, 2, nr. 8: 26 S., 2 Abb., 3 Tab., 1 Tafel. (English summary: The glacial deposits in the cliff of Halkhoved); Kopenhagen (Reitzel).

Madsen, V. \& Nordmann, V. \& Hartz, N. (1908): Eem-Zonerne. - Danm. geol. Unders. Rk. II, 17: 302 S., 27 Abb., Atlas mit Taf. 1-12, 1 K.; (Résumé an francais: La stratigraphie de l'argile à Cyprina islandica.); Kopenhagen (Reitzel).

Madsen, V. (1916): Ristinge Klint. - Danm. geol. Unders. Rk. IV, 1, nr. 2: 32 S., 8 Abb., 2 Tab.; (Résumé en francais: Ristinge Klint.); Kopenhagen (Reitzel).

Madsen, V. (Hrsg.) (1928 a): Übersicht über die Geologie von Dänemark. - Danm. geol. Unders. Rk. V, 4: 216 S., 16 Abb., 2 Taf.; Kopenhagen (Reitzel).

- (1928 b): Führer für die Exkursionen in Dänemark der Internationalen Geologen-Versammlung zu Kopenhagen 1928. - Danm. geol. Unders. Rk V, 5: 112 S., 35 Abb., 1 Kt., Kopenhagen (Reitzel).

Marcussen, I. (1977): Deglaciation landscapes formed during the wasting of the late Middle Weichselian ice sheet in Denmark. - Danm. geol. Unders. Rk. II, 110: 72 S., 14 Abb.; (Dansk sammendrag: Deglaciations landskaber dannet under smeltningen af isskjoldet $\mathrm{i}$ sen mellem weichsel i Danmark); Kopenhagen (Reitzel).

RosenKrantz, A. (1944): Nye bildrag til forstaaelsen af Ristinge Klints opbygning. - Meddr. dansk geol. Foren. 10: 431-435, 2 Abb.; Kopenhagen (Reitzel).

SJörring, S. (1973): Some problems in the till stratigraphy of the northeastern part of Sjaelland. - Bull. geol. Inst. Univ. Uppsala, N.S., 5: 31-35, 5 Abb., 1 Tab.; Stockholm (Almqvist \& Wiksell).

- (1974): Klinterne ved Hundested. - Meddr. dansk geol. Foren., Aarsskrift for 1973: 108117,4 Abb.; Kopenhagen (Reitzel).

- (1977 a): Glacialtektonik og istidsgeologi. - Dansk Natur-Dansk Skole, Aarsskrift 1977: 31-44, 9 Abb.; Kopenhagen.

- (1977 b): The glacial stratigraphy of the island of Als, southern Denmark. - Z. Geomorph. N.F. Suppl.-Bd., 27: 1-11, 7 Abb.; Berlin - Stuttgart (Borntraeger). 